Research Article

\title{
Composite Plate Phased Array Structural Health Monitoring Signal Reconstruction Based on Orthogonal Matching Pursuit Algorithm
}

\author{
Yajie Sun, ${ }^{1,2,3}$ Feihong Gu, ${ }^{1,3}$ Sai Ji, ${ }^{1,2,3}$ and Lihua Wang ${ }^{4}$ \\ ${ }^{1}$ Jiangsu Engineering Centre of Network Monitoring, Nanjing University of Information Science and Technology, \\ Nanjing 210044, China \\ ${ }^{2}$ Jiangsu Collaborative Innovation Center on Atmospheric Environment and Equipment Technology, \\ Nanjing University of Information Science and Technology, Nanjing 210044, China \\ ${ }^{3}$ School of Computer and Software, Nanjing University of Information Science and Technology, Nanjing 210044, China \\ ${ }^{4}$ School of Information and Control, Nanjing University of Information Science and Technology, Nanjing 210044, China \\ Correspondence should be addressed to Yajie Sun; syj@nuist.edu.cn
}

Received 25 July 2017; Accepted 23 October 2017; Published 19 December 2017

Academic Editor: Haoran Xie

Copyright @ 2017 Yajie Sun et al. This is an open access article distributed under the Creative Commons Attribution License, which permits unrestricted use, distribution, and reproduction in any medium, provided the original work is properly cited.

\begin{abstract}
In order to ensure the safety of composite components, structural health monitoring is needed to detect structural performance in real-time at the early stage of damage occurred. This is difficult to detect complex components with single sensor detection technology, so that ultrasonic phased array technology using multisensor detection will be selected. Ultrasonic phased array technology can scan the structure in all directions and angles without moving or less moving the probe and becomes the first choice of structural health monitoring. However, a large amount of data will be generated when using ultrasonic phased array with Nyquist sampling theorem for structural health monitoring and is difficult to storage, transmission, and processing. Besides, traditional Nyquist sampling cannot satisfy the sampling of large amounts of data without distortion, so a more efficient acquisition technique must be chosen. Compressive sensing theory can ensure that if the signal is sparse, it can be sampled in low sampling rate which is much less than two times of the sampling rate as defined by Nyquist sampling theorem for a large number of data and reconstructed in high probability. Then, the experiment result indicated that the orthogonal matching pursuit algorithm can reconstruct the signal completely and accurately.
\end{abstract}

\section{Introduction}

Structural health monitoring is an important technology on the performance of structure and damage of detection $[1,2]$. It uses the sensor technology and advanced signal processing method, in the early time when structural damage occurred, for real-time monitoring of response of the detected structure and properties of the target system. Then, the structure damage can be identified timely and accurately. Along with the continuous development of aviation and aerospace, aircraft structure design is ever-changing. The application requirement of aircraft material, which is light and effective, is also increased dramatically. So the composite materials with the characteristics of light mass and high strength are widely used in the fields of aviation and aerospace. However, in the forming process of the composites, the damages like defects of crack, doping, air bubble, and so on are greatly influenced by the difference of the manufacturing process and the influence of various artificial factors. In order to ensure the safety of the performance of the composite products, it is essential to use the efficient structural monitoring technology-the ultrasonic phased array technology.

With its flexible beam deflection and strong focusing performance, ultrasonic phased array technology attracted the attention of researchers [3-5]. Ultrasonic phased array can achieve the high efficiency detection of the structure by using the orderly stack of sound beam emitted from each 
array element and is significant for structural health monitoring. Ultrasonic phased array transducer is designed based on the Huygens-Fresnel principle. The transducer is composed of a plurality of mutually independent piezoelectric wafers in an array. Each piezoelectric wafer is referred as a unit. When multiple independent piezoelectric wafers are arranged by certain shape and size, in order to transmit the signal, the ultrasonic signal superimposed by each unit in the array forms a new wavefront. In the receiving process of the reflected wave, the receiving of the receiver is controlled by a certain rule and time sequence, and the signal synthesis is carried out, and then the synthesis result is displayed in an appropriate form. Compared with the traditional single sensor detecting, the beam scanning of ultrasonic phased array is flexible, the detection speed is faster, the resolution is higher, and the detection of complex parts is more suitable. In recent years, the research of ultrasonic phased array detection has been developed both at home and abroad [6-9]. The advanced ultrasonic phased array is also used in testing equipment in the field of aviation and space. However, a large amount of data will be produced when monitoring the composite structure real-time and online by the use of ultrasonic phased array technology with Nyquist sampling theorem, which not only needs complicated processing of the data but also needs higher requirements for the acquisition system to complete the data collection.

Compressive sensing (CS) pointed out that as long as the signal is sparse, it can complete the signal acquisition with the much lower sampling rate than the traditional Nyquist sampling theorem [10, 11]. At first, a suitable orthogonal sparse transform basis will be chosen to reduce the number of nonzero values in the sparse coefficient vector in order to get the signal sparse representation. Secondly, in order to achieve a high probability of recovering the original signal, it is necessary to construct a projection observation matrix to make a projection transform for the signal. During this process, the matrix must not be related to the sparse transform basis and need to meet the restricted isometry property at the same time. Finally, a reconstruction algorithm which has high stability, low computational complexity, and high reconstruction precision is designed. The theory can alleviate the pressure caused by the storage, transmission, and data processing. Therefore, the proposed reconstruction method based on orthogonal matching pursuit algorithm of phased array structural health monitoring signal is a better solution of the difficulty of dealing with a large amount of data, in the process of ultrasonic phased array of structural health monitoring.

\section{Compressive Sensing}

Compressive sensing is a novel theory of sampling and reconstructing for sparse signal. The traditional Nyquist sampling theorem obtains the data by uniform sampling, and compressive sensing theory can complete the signal acquisition with inner product operation of signal and observation matrix. As long as the original signal is sparse in the

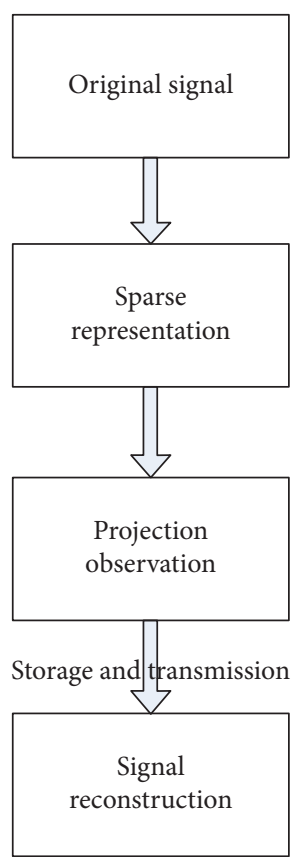

FIgURE 1: The process of compressive sensing.

time domain or the basis of a certain transform, compressive sensing technology can collect the signal with lower sampling rate and reconstruct the signal with high probability. The sampling method is partly collecting the signal information, but not the whole signal, so that it can effectively reduce the complexity of the data. The process of compressive sensing is shown in Figure 1.

There are three main parts of compressive sensing theory, including sparse representation of signal, projection observation of measurement matrix, and sparse reconstruction of signal.

The sparsity of signal is the precondition and foundation of the compressive sensing theory. Thus, the primary task is the sparse representation of signal. Let $\left\{\psi_{i}\right\}_{i=1}^{N}$ is a group basis vector of discrete signal space $R^{N}$ of finite length, and then the signal $x \in R^{N}$ can be linear expressed as follows:

$$
\begin{aligned}
x & =\sum_{i=1}^{N} \theta_{i} \psi_{i} \\
\text { or } x & =\Psi \Theta,
\end{aligned}
$$

where $\Psi=\left[\psi_{1}, \psi_{2}, \ldots, \psi_{N}\right]$ is a transformation matrix with the size of $N \times N . \Theta$ is called the sparse coefficients and is the transform vector of $x$ in $\Psi$ domain. The sparse coefficients satisfy the following expression:

$$
\begin{aligned}
\Theta & =\Psi x \\
\text { or } \theta_{i} & \leq x, \psi_{i} \geq \psi_{i}^{T} x .
\end{aligned}
$$

The core of compressive sensing theory is the design of the measurement matrix, which directly determines whether 
the compressive sensing can be successfully implemented. If the signal $x$ has sparse representation in an orthogonal transform basis $\Psi$, the $M$-dimensional linear projection observation vector $y$ can be expressed as

$$
y=\Phi \Theta=\Phi \Psi^{T} x
$$

where $\Phi \in R^{M \times N}$ is a measurement matrix and is not related to the transform basis $\Psi$.

Sufficient information about the signal $x$ is included in these little values, and if $\Xi=\Phi \Psi^{T}$, (3) can be converted to

$$
y=\Phi \Psi^{T} x=\Xi x
$$

where $\Xi$ is the sensing matrix.

Signal reconstruction is the inverse problem relative to compressed sampling and can reconstruct the signal $x$ from observational value $y$. The reconstructed signal can be obtained by solving (4). The problem is less definite and has infinitely many solutions. Candes and Tao proved that the solution of the equation can be solved by solving the minimum $l_{0}$-norm.

$$
\min \|\Theta\|_{0} \quad \text { s.t. } \quad y=\Phi \Theta=\Phi \Psi^{T} x .
$$

It is a linear programming problem, and it is also a convex optimization problem. If the reconstruction error is taken into account, (5) can be converted to a minimum $l_{1}$-norm problem:

$$
\min \|\Theta\|_{1} \quad \text { s.t. } \quad\|\Phi \Theta-y\|_{2} \leq \varepsilon
$$

\section{Reconstruction of Ultrasonic Phased Array Signal}

The phased array signal with continuous amplitude is collected by piezoelectric sensor array, with the principle of ultrasonic phased array. The large amount of signal is difficult to be stored, transmitted, and processed. In order to facilitate data processing, the phased array signal that has sparse representation can be obtained, by selecting the appropriate sparse transform basis to reduce the nonzero values in sparse coefficient vector.

3.1. Sparse Representation Based on Fourier Transform. In the sparse representation process of ultrasonic phased array signal, the signal will be projected onto the orthogonal transform matrix. The commonly used orthogonal transform basis includes the discrete cosine transform basis, Fourier transform basis, wavelet basis, and curvelet basis [12-15].

Fourier transform can express a function that satisfies certain condition as a trigonometric function or a linear combination of their integration. In different research fields, it includes continuous Fourier transform, discrete Fourier transform, and a variety of different Fourier transform forms.

Discrete Fourier transform (DFT) is a method that transforms the finite time domain data into the frequency domain data [13]. When a discrete time signal $x_{n}(n=0,1, \ldots, N-1)$ with $N$ sequence values is given, the following transform is the discrete Fourier transform of $x_{n}$ :

$$
X_{k}=\sum_{n=0}^{N-1} x_{n} \exp \left(-j \frac{2 \pi n k}{N}\right), \quad k=0,1, \ldots, N-1
$$

And the inverse transform is the inverse discrete Fourier transform.

$$
x_{n}=\frac{1}{N} \sum_{k=0}^{N-1} X_{k} \exp \left(j \frac{2 \pi n k}{N}\right), \quad n=0,1, \ldots, N-1
$$

3.2. Projection Observation of Phased Array Signal. In order to achieve a high probability of reconstructing the original signal, compressive sensing needs a projection observation matrix $\Phi$ to complete the projection transform that is not related to sparse transform basis $\Psi$ and satisfies the restricted isometry property. Recently, there are some measurement matrices that satisfy the restricted isometry principle, including the Gauss random matrix, Bernoulli random matrix, Hadamard matrix, Toeplitz matrix, and random Fourier matrix [16-19]. Although these measurement matrices can make a high probability of satisfying the restricted isometry principle, it still has some shortcomings in practical application, such as computational complexity and storage difficulty. Gauss matrix is not only related to most fixed orthogonal basis but also satisfies the restricted isometry property, so the Gauss matrix can be used as the projection observation matrix $[18,19]$. For the ultrasonic phased array signal, the Gauss random measurement matrix is multiplied with the sparse coefficient, and then the observation vector of the signal can be obtained.

Let the measurement matrix $\Phi$ is $M \times N, \Phi \in R^{M \times N}$, and the general term can be displayed as follows:

$$
\Phi(i, j)=\frac{1}{\sqrt{M}} h_{i j}
$$

Each element of the matrix is independent to the Gaussian distribution. The mean is 0 , and the variance is $1 / \sqrt{M}$. This matrix is not related to the vast majority of sparse signals and requires less measurement values in reconstructing signal. Gauss random measurement matrix is a very flexible matrix with the disadvantage of high uncertainty. A signal with a length of $N$ and a sparse degree of $K$ only needs $M \geq c K \log (N / K)$ as measured values to recover the original signal with high probability, where $c$ is a very small constant.

\subsection{Signal Reconstruction Based on Orthogonal Matching} Pursuit Algorithm. The ultimate goal of compressive sensing theory is to efficiently reconstruct the original signal. And in signal reconstruction process, it is necessary to find the sparsest solution of the equation $y=\Phi \Psi x$. The former compressive sensing reconstruction algorithm is divided into two categories. One kind of reconstruction algorithm is convex 
optimization algorithm [20]. These algorithms mainly increase the constraint to obtain the sparsest solution. The norm bound form is expressed as follows:

$$
\min \|\alpha\|_{p}, \quad \text { s.t. } \quad y=\Phi \Psi \alpha,
$$

where $p \in R,\left(\|\alpha\|_{p}\right)^{p}=\sum_{i=1}^{N}\left|\alpha_{i}\right|^{p}$. The common algorithms are the base pursuit algorithm (BP) based on the linear programming and the gradient projection sparse algorithm (GPSR). The complexity of the algorithm is mostly determined by the number of iterations required by finding the right support set. Therefore, the computing speed of the algorithm is fast, but the need of measurement data is more and the precision is low. The other kind of reconstruction algorithm is greedy iterative algorithm [21-23]. This kind of algorithm mainly uses the combination of local optimization to find the nonzero coefficient to obtain the original signal. The common algorithms are matching pursuit algorithm (MP) and orthogonal matching pursuit algorithm (OMP). The computing speed of the algorithm is low, but the need of measurement data is less and the precision is high.

In the process of the reconstruction of phased array signal, the reconstruction is transformed into a convex optimization problem with minimum $l_{1}$-norm, and then the $l_{1}$-norm is approximated to a linear programming and quadratic programming problem.

OMP is an improved algorithm of MP. This algorithm followed by the MP in the selection of the best atomic criteria improves the shortage of only guaranteeing the orthogonality between the residual error and the best atom during the each iteration. Before the signal projection, OMP will make all traverse atoms orthogonal and then make projection for the signal on the orthogonal atomic space. The components and residual of the signal on the orthogonal atomic space will be obtained. OMP ensures that the residuals will orthogonal with the selected atoms after each iteration.

\section{Experiment and Results}

In the experiment, nine piezoelectric elements in the linear array are arranged in the composite plate. The diameter and the thickness of the piezoelectric element are $8 \mathrm{~mm}$ and $0.48 \mathrm{~mm}$, respectively. And the distance between two adjacent piezoelectric elements is $12 \mathrm{~mm}$. Data collection points are 1024, and the sampling frequency is $f_{s}=1000000 \mathrm{~Hz}$.

The whole process makes one array element as a drive transmitting signal and makes the other eight elements as sensors to receive the reflection signal. Nine array elements stimulate the signal in turns, and each degree corresponds to 72 signals. Then, $9 \times 8 \times 181$ signals can be obtained after monitoring from 0 degrees to 180 degrees by phased array scanning for composite plate structure. Here, we selected the data received by array element number 1 at the direction of 90 degrees which is emitted by array element number 0 , and other degree processing method is consistent. The waveform of the data in time domain is shown in Figure 2.

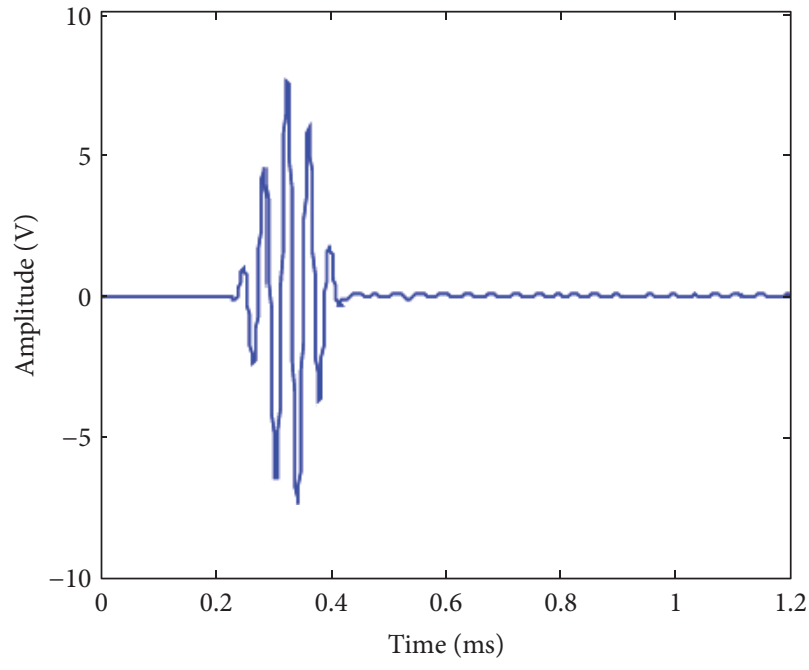

FIgURE 2: The original signal in time domain.

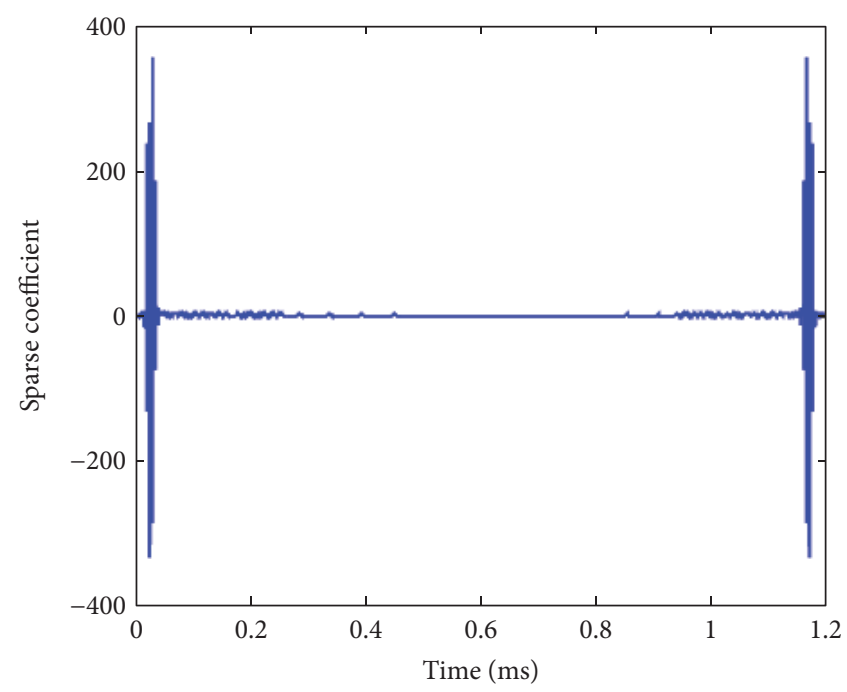

Figure 3: The sparse coefficient after DFT.

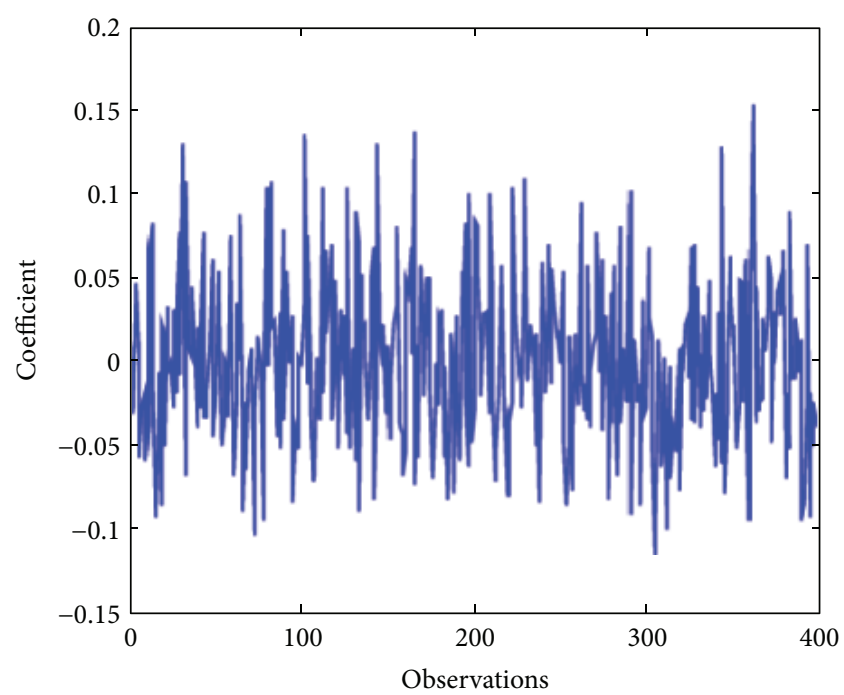

FIGURE 4: The signal observed by Gauss matrix. 


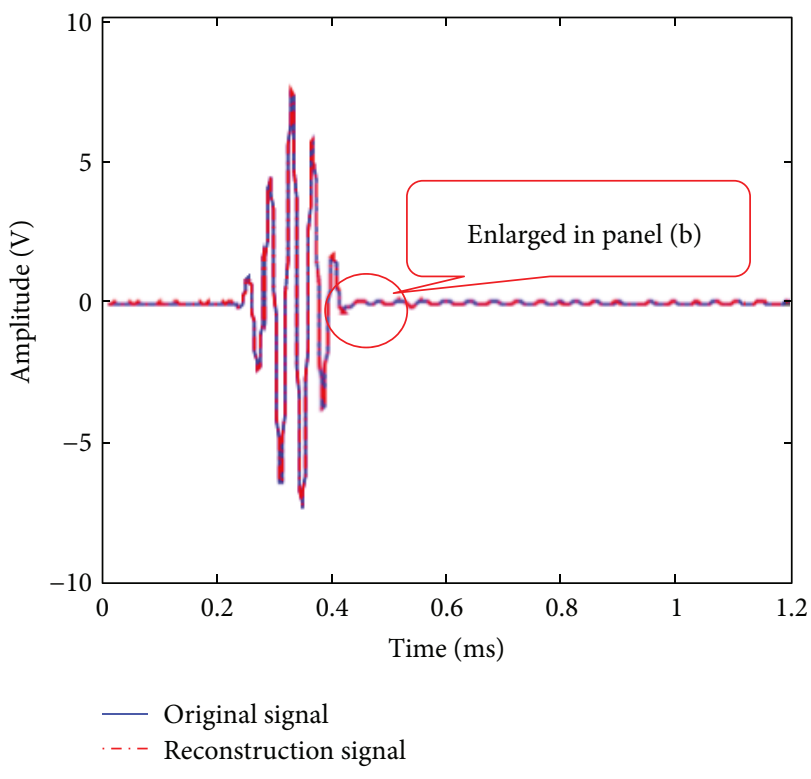

(a)

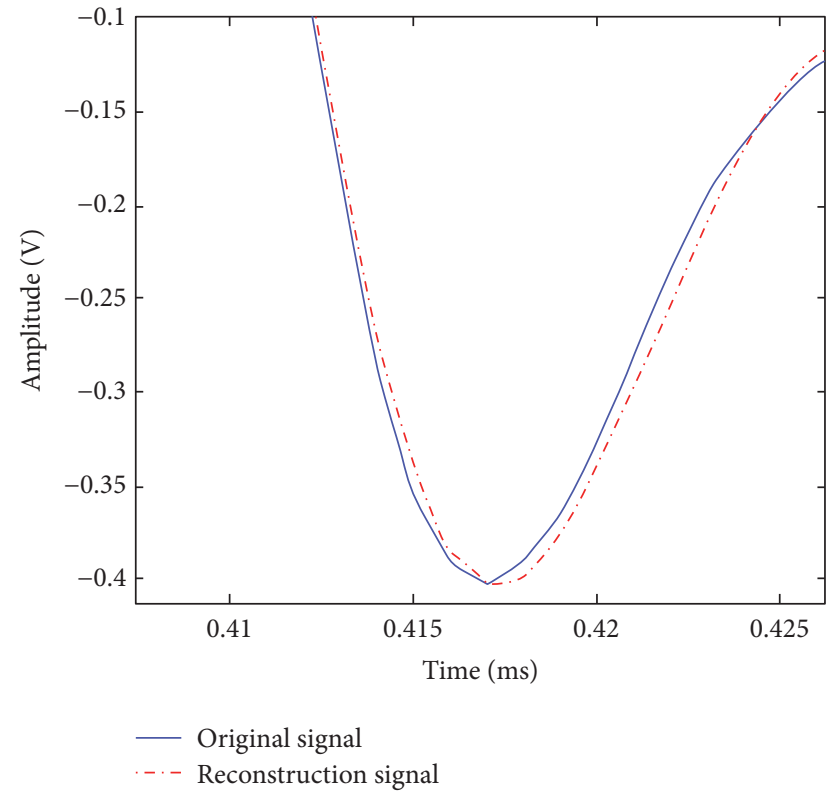

(b)

FIgURE 5: Reconstruction based on orthogonal matching pursuit algorithm.

The discrete Fourier transform is used for processing the received signal, and the sparse representation of the original signal will be obtained as been shown in Figure 3. It can be seen from the figure that the sparse coefficient of the phased array signal after DFT is mostly equal or close to zero, which is consistent with the feature of the sparse signal.

Then, we select the sparse signal to complete the projection observation, where the length of ultrasonic phased array data is $N=1200$, and the number of observations is $M=400$. The result is shown in Figure 4.

Finally, the orthogonal matching pursuit algorithm is used for signal reconstruction. And the result is shown in Figure 5. In Figure 5(a), because the reconstructed signal of the orthogonal matching pursuit algorithm is very close to the original signal, it is difficult to distinguish in the waveform. Therefore, the local waveform is magnified, as shown in Figure 5(b).

Compared with the original phased array signal, the reconstructed signal obtained by the orthogonal matching pursuit algorithm has some differences in the signal waveform. In order to more accurately determine the effect of the reconstruction algorithm, the reconstruction error will be generated by the numerical display, as shown in Figure 6 . The overall reconstruction error is within $0.15 \%$, most of which is almost close to zero.

In order to make a clearer comparison of the effectiveness of the reconstruction algorithm, two reconstruction errors are used to compare the reconstruction error in a quantitative way.

The absolute error and the relative error are calculated, respectively.

$$
\delta=\left|x_{1}-x\right|
$$

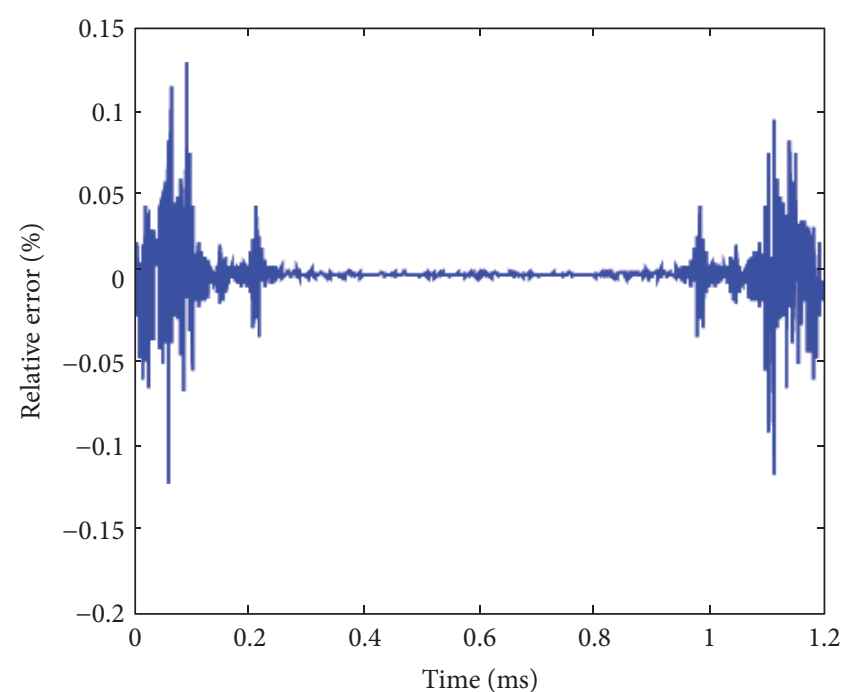

FIGURE 6: Figures of reconstruction error.

$$
\Delta=\frac{\left|x_{1}-x\right|}{x} \times 100 \%
$$

where $x_{1}$ is the reconstructed signal and $x$ is the original signal.

Table 1 shows the error comparison of reconstruction algorithms.

\section{Conclusion}

The composite plate phased array structural health monitoring signal reconstruction method based on orthogonal matching pursuit algorithm can effectively solve the 
TABLE 1: The error comparison of reconstruction algorithms.

\begin{tabular}{lcc}
\hline $\begin{array}{l}\text { Error } \\
\text { Algorithm }\end{array}$ & $\delta / \mathrm{V}$ & $\Delta / \%$ \\
\hline OMP & 2.7320 & 0.13 \\
MP & 35.1390 & 2.75 \\
BP & 27.4349 & 1.66 \\
GPSR & 52.4268 & 5.82 \\
\hline
\end{tabular}

difficulty of data storage and processing, due to the huge amount of data produced by the ultrasonic phased array. Firstly, the discrete Fourier transform is used for sparse representation to reduce the nonzero values of the signal. Secondly, the Gauss matrix is used for projection observation to obtain the observations. Thirdly, the orthogonal matching pursuit algorithm is used for sparse reconstruction to approximate the original signal. The experiment shows that the orthogonal matching pursuit algorithm can be used to reconstruct the phased array signal monitored by the structure of the composite plate in less mistakes. In future research, we can choose more optimized projection observation matrix and more efficient reconstruction algorithm to reconstruct the ultrasonic phased array signal more efficiently.

\section{Conflicts of Interest}

The authors declare that they have no conflicts of interest.

\section{Acknowledgments}

This work is supported by the National Natural Science Foundation of China (Grant nos. 51405241 and 61672290), the Jiangsu Government Scholarship for Overseas Studies, and the PAPD fund.

\section{References}

[1] H. Cao, S. K. Thakar, M. L. Oseng et al., "Development and characterization of a novel interdigitated capacitive strain sensor for structural health monitoring," IEEE Sensor Journal, vol. 15, no. 11, pp. 6542-6548, 2015.

[2] A. Bulletti, P. Giannelli, M. Calzolai, and L. Capineri, "An integrated acousto/ultrasonic structural health monitoring system for composite pressure vessels," IEEE Transactions on Ultrasonics, Ferrorlectrics, and Frequency Control, vol. 63, no. 6, pp. 864-873, 2016.

[3] C. Yuan, C. Xie, L. Li, F. Zhang, and S. M. Gubanski, "Ultrasonic phased array detection of internal defects in composite insulators," IEEE Transactions on Dielectrics and Electrical Insulation, vol. 23, no. 1, pp. 525-531, 2016.

[4] Y. J. Sun, Y. Gao, Y. H. Xue, Y. Cai, and G. Xiang, "Experimental research on phased array ultrasonic monitoring imaging and histogram matching based image enhancement," Chinese Journal of Sensors and Actuators, vol. 28, no. 1, pp. 87-92, 2015.
[5] C. Diego, A. Jiménez, Á. Hernández, C. J. Martín-Arguedas, and C. G. Fernández, "Improved ultrasonic phased array based on encoded transmissions for obstacle detection," IEEE Sensor Journal, vol. 15, no. 2, pp. 827-835, 2015.

[6] B. Puel, D. Lesselier, S. Chatillon, and P. Calmon, "Optimization of ultrasonic arrays design and setting using a differential evolution," NDT \& E International, vol. 44, pp. 797-803, 2011.

[7] Y. Luo, H. Qiu, W. G. Zhang, and Z. P. Wang, "Research on technology of high integration ultrasonic phased array transmission system based on FPGA," Instrument Technique and Sensor, vol. 2, pp. 26-28, 2014.

[8] S. W. Liu and Y. Luo, "Data acquisition system for ultrasonic phased array system based on embedded NiosII," Instrument Technique and Sensor, vol. 6, pp. 72-75, 2014.

[9] Z. G. Zhou, Y. Li, F. H. Chen, and W. Zhou, "Research on three dimensional imaging method using ultrasonic matrix array transducer," Chinese Journal of Scientific Instrument, vol. 37, no. 2, pp. 371-378, 2016.

[10] D. L. Donoho, "Compressed sensing," IEEE Transactions on Information Theory, vol. 52, no. 4, pp. 1289-1306, 2006.

[11] S. Friedland, Q. Li, and D. Schonfeld, "Compressive sensing of sparse tensors," IEEE Transactions on Image Processing, vol. 23, no. 10, pp. 4438-4447, 2014.

[12] Z. Xu, "Detection algorithm for low SNR signal in UWB systembased on sparse wavelet transform," Chinese Journal of Scientific Instrument, vol. 34, no. 4, pp. 825-830, 2013.

[13] S. Liu, T. Shan, R. Tao et al., "Sparse discrete fractional Fourier transform and its applications," IEEE Transactions on Signal Processing, vol. 62, no. 24, pp. 6582-6595, 2014.

[14] L. Cao, L. Jin, H. Tao, G. Li, Z. Zhuang, and Y. Zhang, "Multifocus image fusion based on spatial frequency in discrete cosine transform domain," IEEE Signal Processing Letters, vol. 22, no. 2, pp. 220-224, 2015.

[15] P. Pedram and U. Michael, "Optimality of operator-like wavelets for representing sparse $\mathrm{AR}(1)$ processes," IEEE Transactions on Signal Processing, vol. 63, no. 18, pp. 48274837, 2015.

[16] E. Candes, "The restricted isometry property and its implications for compressed sensing," Comptes Rendus Mathematique Academie Des Sciences, vol. 346, no. 9-10, pp. 589-592, 2008.

[17] Y. J. Sun and F. H. Gu, "Compressive sensing of piezoelectric sensor response signal for phased array structural health monitoring," International Journal of Sensor Networks, vol. 23, no. 4, pp. 258-264, 2017.

[18] E. Candes and T. Tao, "Decoding by linear programming," IEEE Transactions on Information Theory, vol. 51, no. 12, pp. 4203-4215, 2005.

[19] H. L. Shi, H. Zhang, G. Li, and X. Wang, "Stable embedding of Grassmann manifold via Gaussian random matrices," IEEE Transactions on Information Theory, vol. 61, no. 5, pp. 29242941, 2015.

[20] J. J. Feng, G. Zhang, and F. Q. Wen, "Improved sparse signal reconstruction algorithm based on SL0 norm," Journal of Data Acquisition and Processing, vol. 31, no. 1, pp. 178-183, 2016.

[21] H. Wu and S. Wang, "Adaptive sparsity matching pursuit algorithm for sparse reconstruction," IEEE Signal Processing Letter, vol. 19, no. 8, pp. 471-474, 2012. 
[22] M. R. Yang and F. D. Hoog, "Orthogonal matching pursuit with thresholding and its application in compressive sensing," IEEE Transactions on Signal Processing, vol. 63, no. 20, pp. 5479-5486, 2015.

[23] S. K. Sahoo and A. Makur, "Signal recovery from random measurements via extended orthogonal matching pursuit," IEEE Transactions on Signal Processing, vol. 63, no. 10, pp. 2572-2581, 2015. 


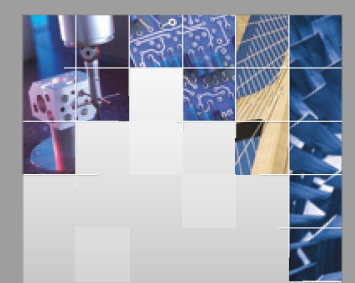

\section{Enfincering}
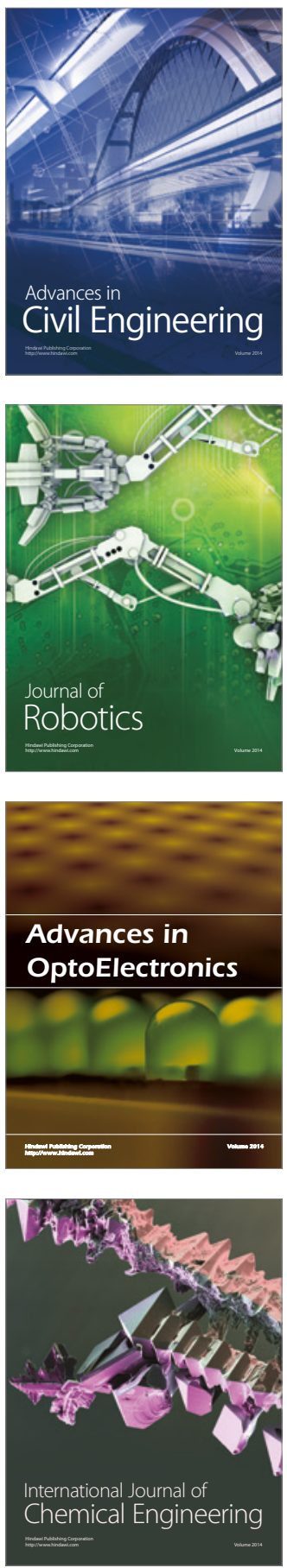

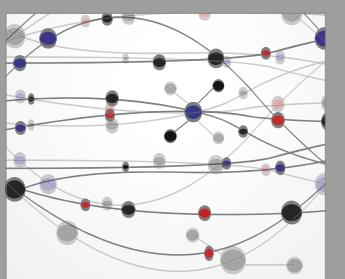

The Scientific World Journal

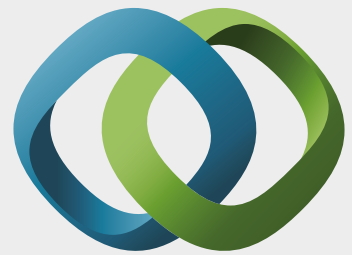

\section{Hindawi}

Submit your manuscripts at

https://www.hindawi.com
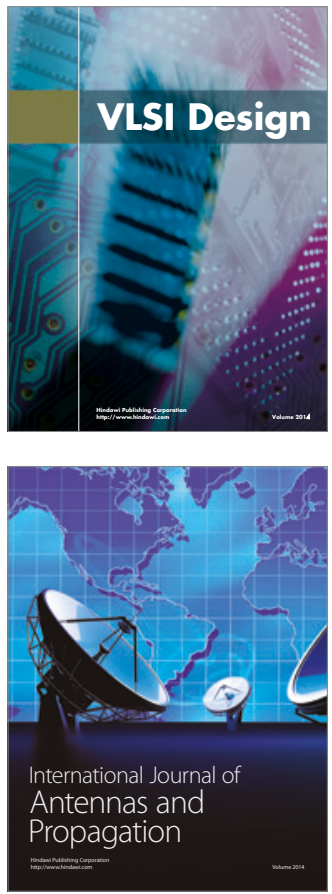

\section{Rotating}

Machinery
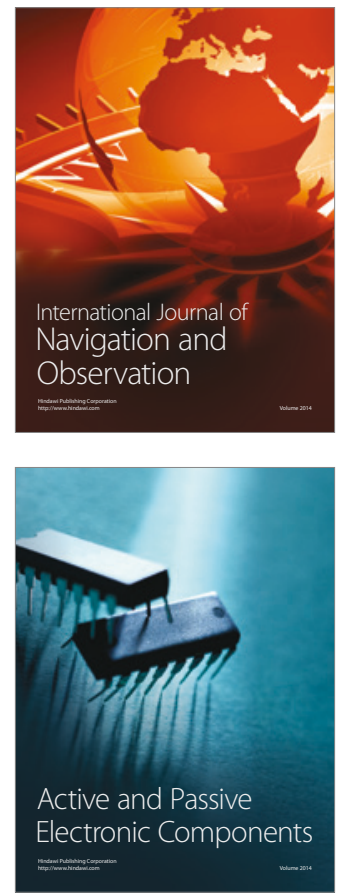
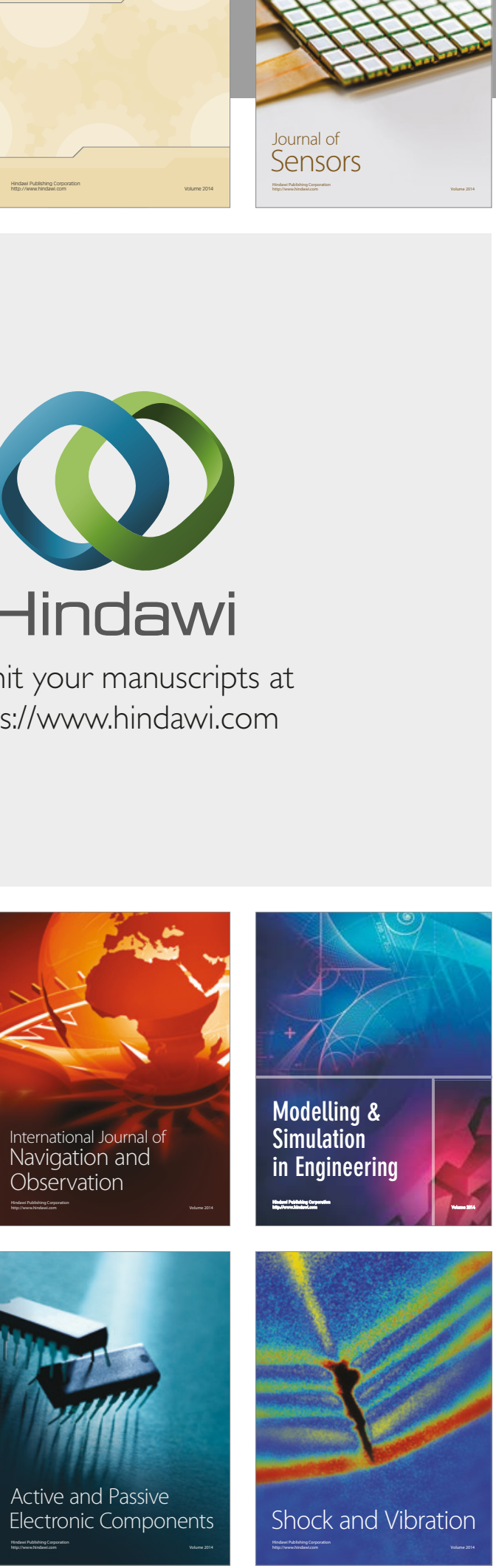
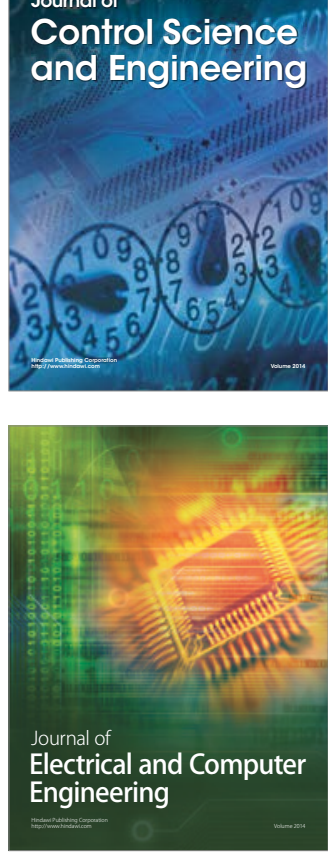

Distributed

Journal of

Control Science

and Engineering
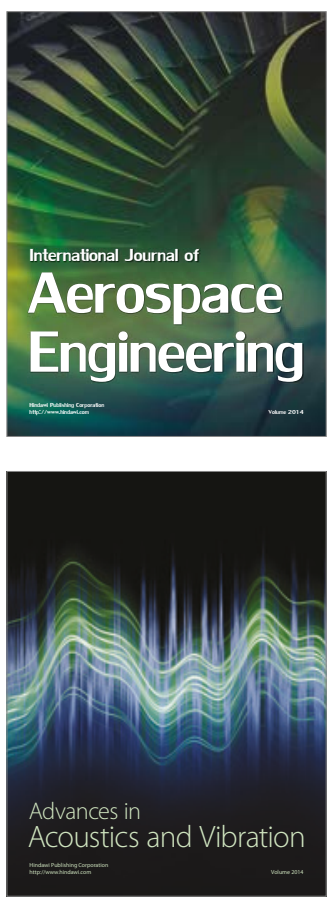

Sensor Networks 